Research Article

\title{
Madanpal Nighantu (Lexicon): A Critical Analysis
}

\author{
Deepak Verma', Kamini Kaushal ${ }^{2}$, Ashwini Kumar Sharma ${ }^{3}$, Mahesh Dixit ${ }^{4}$
}

${ }^{1}$ PG Scholar, ${ }^{2}$ Professor \& H.O.D., ${ }^{3}$ Associate Professor, ${ }^{4}$ Principal \& Professor, PG Department of Dravyaguna Vijnana, MMM Government, Ayurved College, Udaipur, Rajasthan.

DOI: https://doi.org/10.24321/2394.6547.201902

\section{I $\quad \mathbf{N} \quad \mathbf{F} \quad \mathbf{O}$}

\author{
Corresponding Author: \\ Deepak Verma, PG Department of Dravyaguna \\ Vijnana, MMM Government, Ayurved College, \\ Udaipur, Rajasthan. \\ E-mail Id: \\ dr.deepakverma3@gmail.com \\ Orcid Id: \\ https://orcid.org/0000-0001-7622-106X \\ How to cite this article: \\ Verma D, Kaushal K, Sharma AK et al. Madanpal \\ Nighantu (Lexicon): A Critical Analysis. J Adv \\ Res Ayur Yoga Unani Sidd Homeo 2019; 6(1\&2): \\ 10-19.
}

Date of Submission: 2019-06-24

Date of Acceptance: 2019-07-11

\section{$\begin{array}{llllllll}\mathbf{A} & \mathbf{B} & \mathbf{S} & \mathbf{T} & \mathbf{R} & \mathbf{A} & \mathbf{C} & \mathbf{T}\end{array}$}

Ayurveda, the ancient traditional science is the treasure of India. The great sages like Charaka and Sushruta have elaborated various available herbs in their respective periods in their texts. The categorization of then available herbs into Ganas or Mahakashya varga depending on their chief action/drug has immensely contributed in clinical field. The texts have served the foundational reference to many lexicon. These lexicons i.e. Ashtang nighantu, Dhanwantari nighantu, Raj nighantu, Bhavpraksh nighantu are simplified texts exclusively for the study of plants and its medicinal properties and the codify plant names by grouping synonyms of one plant together. Madanpal nighantu is one such available lexicon the dates back to 11-13 cen. A.D. This article emphasizes mainly on distinctive feature of Madanpal Nighantu. This lexicon followed by the ancient texts like Sushruta Samhita; Ashtang Nighantu; Dravyaguna Samngrha and Shabda pradipa.

Keywords: Madanpal Nighantu, Lexicon, Ayurveda

\section{Inroduction}

Madanapala Nighantu is also known as Madana Vinod. Going through the inclusion materials of the text is to be composed in a very precise form (neither too large nor small) consisting whole of the materials related with plant materia medica. On this background the text has been written in 1374 A.D. (1431 Vikram samvat) in the month of/magha the sixth day- monday. ${ }^{1}$

The text is divided into the following 13 sections: ${ }^{2}$

- Abhayadi

- Shuntyadi

- Karpuradi

- Suvarnadi

- Vatadi

- Phaladi/Drakshadi

- Shaaka

- Paaniyadi

- Ikshukadi
- Dhanya Varga

- Dhanyakratannadi Varga

- Mamsa Varga

- Mishraka Varga

Among plant drugs Ahiphena (Opium) has been described (because China is cultivating at that time), but not Yashada=Zinc(because zinc is producing at that time in rajasthan's zawar mines area). In Mishraka Varga no drug has been described, where indications regarding Anupan and Seasonal conduct have been mentioned for healthy life.

The following drugs are specially mentioned in Madanpal Nighantu are:

- Bhang

- Kankushta

- Jayapaala

- ParasikaYavani

- Chauhara

- Maarjaari 
- Sinduri

- Silomaani

- Kharjura

- Kharbuza

- Amruthaphala

- Baadaam

- Anjeera

- Madhukarkati

- Grunjanakha

- Yavanala

- Kundalika

\section{Aim and Objectives}

Aim

To study the distinctive feature of Madanpal Nighantu.

\section{Objectives}

- To collect the literature on Madanpal Nighantu according to Ayurvedic classics and modern classification.

- To assess the evidence of various types of plant species.

- Possible species; on the basis of basonyms/ synonyms in the text of Madanpal Nighantu.

Identical Feature Based on Sanskrit Synonyms \& Possible Species ${ }^{3}$

\begin{tabular}{|c|c|c|}
\hline Sanskrit Name & Identical Feature & Possible Species \\
\hline $\begin{array}{l}\text { Shaalparni } \\
\text { (sarivan) }^{4}\end{array}$ & $\begin{array}{l}\text { Dhruva, Soumya, Triparni, Peetani, Sthira, } \\
\text { Vidarigandha, Atiguaha, Deerghsmoola, Anshumati. }\end{array}$ & Desmodium triflorum Linn. \\
\hline $\begin{array}{l}\text { Prishnaparni } \\
\quad \text { (pithvan }^{5}\end{array}$ & $\begin{array}{l}\text { Pithvana, Kroshtupuchha, Dhavani, Kalashaaruha, } \\
\text { Srigaalvratta, Ahitiala, Prathhkkaparni, Parnika. }\end{array}$ & Uraria picta (Jacq.) Desv. ex DC. \\
\hline Mashparni6 & $\begin{array}{l}\text { Krishnavranta, Kaamboji, Hayapuchhika, } \\
\text { Maansmaasha, Sinhmukhi, Swadumaasha, Mahasaha. }\end{array}$ & Teramnus labialis (L.f.) Spreng. \\
\hline Mugdaparni ${ }^{7}$ & $\begin{array}{l}\text { Kshudrasaha, Sooryaparni, Kurangini, Vanaja, Ringini, } \\
\text { Shimbi, Sinhi, Maarjargandhika. }\end{array}$ & Phaseolus trilobus \\
\hline Moorva $^{8}$ & $\begin{array}{l}\text { Devi, Madhurasa, Devasreni, Snigdhaparni, } \\
\text { Prathhakparni, Morati, Peeluparnika. }\end{array}$ & $\begin{array}{l}\text { Marsdenia tenacissima (Roxb.) } \\
\text { Moon/Maerua oblongifolia/ } \\
\text { maerua areneria }\end{array}$ \\
\hline Riddhi ${ }^{9}$ & $\begin{array}{c}\text { Sukhamyuga, Lakshmi, Sidhhi, Sarvajanapriya, } \\
\text { Rishisrishta, Rathanga, Maangalya, Shravani, Vasu, } \\
\text { Yogya, Yugyaa, Tushtirashi. }\end{array}$ & Hebenaria intermedia var. foliosa \\
\hline Vradhhi $^{10}$ & Same & $\begin{array}{l}\text { Habenaria edgeworthii Hook.f.ex } \\
\text { Collet }\end{array}$ \\
\hline Kakaoli11 & $\begin{array}{c}\text { Madhura, Veera, Kayastha, Ksheershuklika, } \\
\text { Dhwanksholi, Vaayasoli, Swaadumanshi, Payasavini. }\end{array}$ & $\begin{array}{l}\text { Fritillaria roylei common } \\
\text { name yellow Himalayan fritillary/ } \\
\text { Roscoea purpurea }\end{array}$ \\
\hline Ksheer kakoli12 & $\begin{array}{c}\text { Suravha, Kshirini, Peevar, Sadrash-Skandha, Sa- } \\
\text { Sugandhaka, Kshirkakolika }\end{array}$ & $\begin{array}{l}\text { Lilium polyphyllum/ roscoea } \\
\text { alpinia/lasia spinosa }\end{array}$ \\
\hline$M{ }^{13} a^{13}$ & Shaalparnidrashya, Medobhava, Dhara, & Polygonatum verticillatum (L.) All. \\
\hline Mahameda ${ }^{14}$ & Vasukshidra, Tridanta, Devtamani. & $\begin{array}{l}\text { Polygonatum cirrhifolium (Wall.) } \\
\text { Royle }\end{array}$ \\
\hline Jeevak ${ }^{15}$ & Madhura, Sringi, Hrasva-Anga, Koorchsheershaka. & $\begin{array}{l}\text { Malaxis acuminate D.Don Syn. } \\
\text { Microstylis wallichii Lindl. }\end{array}$ \\
\hline Rishabhaka ${ }^{16}$ & Veer, Indtaksha, Vishani, Durdhar, Vrash. & $\begin{array}{c}\text { Manilkara hexandra/Microstylis } \\
\text { muscifera Ridley }\end{array}$ \\
\hline Erand $^{17}$ & $\begin{array}{l}\text { Deerghadanda, Taruna, Vardhamanaka, Chitra, } \\
\text { Panchangula, Vyaghrapuchha, Gandharva-Hasta. }\end{array}$ & Ricinus communis $L$. \\
\hline Rakta Eranda ${ }^{18}$ & $\begin{array}{c}\text { Hastikarna, Vyaghra, Vyaghratara, Laghu, Uttanpatra, } \\
\text { Urbu, Vaatavairi, Chanchula. }\end{array}$ & Jatropa curcusa L. \\
\hline Shweta Sariva ${ }^{19}$ & $\begin{array}{c}\text { Sharda. Aasphota, Gopkanya, Pratanika, Gopangngna, } \\
\text { Gopvalli, Latavhaa Kashthasariva. }\end{array}$ & Hemidesmus indicus (L.) R.Br. \\
\hline
\end{tabular}




\begin{tabular}{|c|c|c|}
\hline Krishna Sariva ${ }^{20}$ & $\begin{array}{l}\text { Krishnamooli, Bhadrachanadansariva, Bhadra, } \\
\text { Chandangopa, Chandana, Krishnavalli. }\end{array}$ & Cryptolepis buchananii Burm. \\
\hline Yaas $^{21}$ & $\begin{array}{c}\text { Marudbhava, Ananat, Dirghamoola, Yavasaka, } \\
\text { Balpatra, Samudra, Samudrant, Doormool, Atikantaka, } \\
\text { Dhanvyas, Tamramooli, Duh-Saprsha, Duralabha, } \\
\text { Yaasak, Kachhura, Dhanvyavaasak. }\end{array}$ & $\begin{array}{c}\text { Alhagi maurorum Medik/fagonia } \\
\text { Arabica/fagonia cretica/tragia } \\
\text { involucrate }\end{array}$ \\
\hline Mundi ${ }^{22}$ & $\begin{array}{l}\text { Bhikshu, Parivraaji, Paavani, Tapodhana, Shravani, } \\
\text { Srimati, Mundi, Tikta Sravansheerashaka. }\end{array}$ & Sphaeranthus indicus $L$. \\
\hline Mahamundi ${ }^{23}$ & Lobhneeya, Chhinna, Garnthinika. & Leonotis nepetifolia (L.) R.Br. \\
\hline Apamarga $^{24}$ & $\begin{array}{l}\text { Chinchida, Shikhari, Kinhi, Khar Manjari, Adhah Shalya, } \\
\text { Shekhrika, Pratyakapushpi, Mayurika. }\end{array}$ & Achyranthes aspera $L$. \\
\hline Rakta Apamarga ${ }^{25}$ & Vrantaphal, Vashir, Kapi-Pippali. & Achyranthes lappacea $L$. \\
\hline Danti $^{26}$ & $\begin{array}{c}\text { Gunapriya, Naagdanti, Sheeghranukoolak, Upchitra, } \\
\text { Anukumbhi, Vishlya, Udumberachhda, Aakhuparni, } \\
\text { Vrashaiyarnda, Dravanti, Sarvari, Mushkavha, } \\
\text { Sutasreni, Praytyakasreni, Fanjjika. }\end{array}$ & $\begin{array}{c}\text { Baliospermum } \\
\text { montanum (Willd.) Mull. Arg }\end{array}$ \\
\hline Jayapal27 $^{27}$ & Dantibeeja, Tittirphala. & Croton tiglium L. /jamalgota Buch. \\
\hline Trivrat $^{28}$ & $\begin{array}{l}\text { Kumbha, Aruna, Trystrabhandi, Kootarvahini, } \\
\text { Sarvanubhuti, Trivratta, Triputa, Sarala, Sita. }\end{array}$ & Operculina terpathum $L$. \\
\hline Trivrat kala ${ }^{29}$ & $\begin{array}{c}\text { Kaalmeshi, Kaalparni, Ardhachandrika, Sukhena, } \\
\text { Malvika, Masooravidela. }\end{array}$ & Operculina terpethum $L$. \\
\hline Indravaaruni $i^{30}$ & $\begin{array}{c}\text { Indrahva, Vrashbhakshee, Gavadini, Indravaaru, } \\
\text { Kshudraphala, Vishala, Vrashadini. }\end{array}$ & $\begin{array}{l}\text { Citrullus colocynthis } \\
\text { (L.) Schrad. }\end{array}$ \\
\hline Atyendra Vaaruni ${ }^{31}$ & $\begin{array}{c}\text { Chitraphala, Mahaphala, Aatmaraksha, Naagdanti, } \\
\text { Trapushi, Gajachirbhati, Shwetapushpi, Mragakshi, } \\
\text { Pakshsura, Krimiguaha, Chitradevi. }\end{array}$ & Croton Oblingifolius Roxb. \\
\hline Sehunda ${ }^{32}$ & $\begin{array}{l}\text { Vajratunda, Gandir, Vajrakantaka, Snuhi, Samdugdha, } \\
\text { Asipatra, Vajri, Mahataru. }\end{array}$ & $\begin{array}{l}\text { Euphorbia antiquorum L./ E. } \\
\text { nerifolia }\end{array}$ \\
\hline Hemavha ${ }^{33}$ & $\begin{array}{c}\text { Kanakshiri, Hempushpi, Himawati, Kshirini, } \\
\text { Kanchankshiri, Katuparni, Chikarshani, Tiktadugdha, } \\
\text { Hemvati, Pitadugdha, Himadrika. }\end{array}$ & Euphorbia nerifolia/ ipomea spe. \\
\hline Saatla ${ }^{34}$ & $\begin{array}{c}\text { Virala, Saari, Satphala, Bahuphenaka, Charmakasa, } \\
\text { Phena, Diptaa, Naalika. }\end{array}$ & Euphorbia dracuniculodides Lam. \\
\hline Kiratikta $^{35}$ & $\begin{array}{c}\text { Kirat-Tiktaka, Kairaat, Kiraat, Bhunimba, Raamsenak, } \\
\text { Kiratak, Naipal, Naaditikta, Jwarantaka, Kandtikta, } \\
\text { Ardhatikta, Nidrari, Sannipatha. }\end{array}$ & $\begin{array}{l}\text { Swertia chirayita (Roxb. ex } \\
\text { Fleming)/ S.corymbosa }\end{array}$ \\
\hline Trayaman ${ }^{36}$ & $\begin{array}{c}\text { Suhratraana, Traayanti, Girisanuja, Balbhadra, } \\
\text { Krattraana, Varshak, Traaymaanika. }\end{array}$ & Gentiana kurroo Royle. \\
\hline Ashmantaka $^{37}$ & $\begin{array}{l}\text { Maalukapaatra, Yugmapatra, Amlapatraka, } \\
\text { Shlekshantvak, Ashvayoni, Kushali, Paapnashan. }\end{array}$ & Ficus rumphii BI. \\
\hline Kaanchnaar 38 & $\begin{array}{c}\text { Kaanchnaka, Pakaari, Raktapushpaka, } \\
\text { KOVIDAAR=Kuddal, Kuhali, Kuli, Aasphot, Uddaalak, } \\
\text { Swalpkeshar, Chmari. }\end{array}$ & $\begin{array}{l}\text { bauhinia veriegata/B.gaalpinii/ } \\
\text { Bauhinia purpurea/B. acuminate }\end{array}$ \\
\hline Nirgundi ${ }^{39}$ & $\begin{array}{c}\text { Shwetakusum, Sinduka, Sinduvaar. } \\
\text { NEELASAMBHALU= Bhootkeshi, Neelsinduka, } \\
\text { Pushpaneelak, Shephalika, Sheetbjiru, Vanak, } \\
\text { Anilmanjari. }\end{array}$ & $\begin{array}{l}\text { Vitex nirgundo } L . \\
\text { vitex trifolia } L .\end{array}$ \\
\hline
\end{tabular}




\begin{tabular}{|c|c|c|}
\hline Shatavari ${ }^{40}$ & $\begin{array}{c}\text { Dwipishatru, Dwipaka, Dharkantaka, Naraayani, } \\
\text { Shatapadi, Shatpaat, Bahupatrika. }\end{array}$ & Asparagus recemosus $L$. \\
\hline Maha-Shatavar ${ }^{41}$ & $\begin{array}{c}\text { Urdhvakantha, Peevari, Dheevari, Vari, Abheeru, } \\
\text { Bahupatri, Mahapurushdantika, Sahasraveerya, Keshi, } \\
\text { Tungini, Sukshampatrika. }\end{array}$ & Asparagus sarmentosa Linn \\
\hline Bala $^{42}$ & $\begin{array}{c}\text { Vatyalaka, Sheetpaki, Vatyodarahavya, Bhadrodani, } \\
\text { Samanga, Samansa, Kharyashtika. }\end{array}$ & Sida cordifolia L. \\
\hline Sahdeva ${ }^{43}$ & $\begin{array}{c}\text { Mahabala, Veerpushpi, Brahdbala, Vaatyayani, } \\
\text { Devasaha, Vatya, Peetpushpika. }\end{array}$ & Sida rohmbibolia L. \\
\hline Balaaka $^{44}$ & Atibala, Bharvaaji, Vrakshgandhini. & Ablution indicum $L$. \\
\hline Gangerukit5 & Nagabala, Vishvadeva, Gavekshukaa & Grewia tenex $L$. \\
\hline Raajbala ${ }^{46}$ & $\begin{array}{c}\text { Prasarini, Chruparni, Pratanika, Sarani, Saarani, } \\
\text { Bhadraparni, Suprasara, Saraa. }\end{array}$ & Paederia foetida Linn. \\
\hline Vidarika $a^{47}$ & $\begin{array}{c}\text { Vidarika, Vrakshvalli, Vrakshkanda, Vidalika, } \\
\text { Srangalika, Kandavalli, Swadukanda, Phalashaka. }\end{array}$ & $\begin{array}{l}\text { Pueraria tuberose } \\
\text { (Willd.) DC }\end{array}$ \\
\hline Ksheer - Vidari ${ }^{48}$ & $\begin{array}{c}\text { Shukla, Ksheershukla, Ksheervalli, Payasawani, } \\
\text { Ikshuvalli, Mahashweta, Ksheerkanda, Ikshugandhika. }\end{array}$ & Ipomea digitata $L$. \\
\hline Somavalli49 & Yagyaneta, Somkshiri, Dwijpriya. & $\begin{array}{l}\text { Ephedra gerardiana/serratula } \\
\text { anthelmintica/sarcostemma } \\
\text { acidum var. brevistigma }\end{array}$ \\
\hline Aakshavalli50 & Amarvalli, Khwalli. & $\begin{array}{l}\text { Cassytha filiformis Linn. } \\
\text { Cuscuta chinensis Lam. } \\
\text { Cuscuta reflexa roxb. }\end{array}$ \\
\hline Vatapatri $^{51}$ & Mohini, Deepani, Rechani. & $\begin{array}{c}\text { Kalanchoe pinnata/bryophyllum } \\
\text { pinnatum/bryophyllum calycinum/ } \\
\text { trianthema monogyna }\end{array}$ \\
\hline Lajjalu $^{52}$ & $\begin{array}{l}\text { Mohini, Sprakka, Khadiri, Gandhakarini, Namaskari, } \\
\text { Shamipatri, Samnga, Raktapadika. }\end{array}$ & Mimosa pudica $L$. \\
\hline Moosali53 & $\begin{array}{c}\text { Khalani, Taalpatri, Kaanchanpushpika, Mahavraksha, } \\
\text { Vrakshakanda, Khajoori, Taalmoolika. }\end{array}$ & $\begin{array}{c}\text { Asparagus adscence L./Curculingo } \\
\text { orchioides Gaertn. }\end{array}$ \\
\hline Brahmi $^{54}$ & Saraswati, Soma, Satyavha, Brahmacharinni. & Bacopa monnieri $L$. \\
\hline Brahma Mandooki55 & $\begin{array}{c}\text { Mandookparni, Mandooki, Twashti, Divya, } \\
\text { Mahaushadhi, Kapotavitaka, Munika, Laavnya, } \\
\text { Somvallari. }\end{array}$ & $\begin{array}{l}\text { Centella asiatica } \\
\quad \text { (L.) Urban }\end{array}$ \\
\hline Vaami $^{[56]}$ & Shankhdhara, Vaaribrahmi, Hilmochika. & Enhydra fluctuans \\
\hline Vellentara $^{57}$ & Deerghapatra, Veerdru, Bahuvaarak. & $N A$ \\
\hline Vandaak $^{58}$ & $\begin{array}{l}\text { Vraksharuha, Shekhari, Kaamvrakshak, Vrakshadani, } \\
\text { Kaamtaru, Kaamini, Aapadrohini. }\end{array}$ & $\begin{array}{l}\text { Vanda tessellate/ } v \text {. roxbughii/ } \\
\text { soymida febrifuga }\end{array}$ \\
\hline Aprajita $^{59}$ & $\begin{array}{c}\text { Shwetapushpa, Katabhi, Girikarnika, Sita, Aprajita, } \\
\text { Shweta, Vishghani, Mehanashini. } \\
\text { Vishnukranta, Neelpushpi, Jaya, Vashya, Neelsynda, } \\
\text { Vyaktagandha, Neelpushpa, Gavadini. }\end{array}$ & Clitoria ternetia $L$. \\
\hline Aaramsheetala $^{60}$ & Devi, Gandha Kukkurmardan. & Blumea balsamifera L. DC. \\
\hline Kukkuradru ${ }^{61}$ & $\begin{array}{c}\text { Taamrachood, Sukshmapatra, Mraduchhada, } \\
\text { Kukuronda. }\end{array}$ & Blumea lacera L. DC. \\
\hline
\end{tabular}




\begin{tabular}{|c|c|c|}
\hline Doorva $^{62}$ & $\begin{array}{c}\text { Green=Shasya, Sheetkari, Golomi, Shataparvika. } \\
\text { Shweta Doorva=Shweta, Shwetadanda, Bhargavi, } \\
\text { Durmata, Rooha. }\end{array}$ & Cynodon dactylon $L$. \\
\hline Gandadoorva ${ }^{63}$ & Matsyagandha, Matsyakshi, Shakuladani. & Alternanthera sessils L. (R.Br.) \\
\hline Toyapippali[64] & Ambuvalli, Paloor, Kanchat. & Phyla nodiflora L. Greene \\
\hline Chavya ${ }^{65}$ & Chavan, Uchshishta, Kolvallika. & Piper chaba L. \\
\hline Gajapippali $^{66}$ & Hastimagdha, Gajapippali, Gajakrishna. & $\begin{array}{l}\text { Scindapsus officinalis (Roxb.) } \\
\text { Schott }\end{array}$ \\
\hline Shatapushpa ${ }^{67}$ & $\begin{array}{c}\text { Shatavha, Kaaravi, Mishi, Avaakpushpi, } \\
\text { Tvachi-Ch-Chhitra. } \\
\text { Shetika, Maagadhi. }\end{array}$ & $\begin{array}{l}\text { Pimpinella anisum L./anthum } \\
\text { sowa }\end{array}$ \\
\hline Mishreya $^{68}$ & Mishishaleen, Shaali, Sheet-Shiva. & Foeniculum vulgare $L$. \\
\hline $\begin{array}{l}\text { Ajmoda/sthool } \\
\text { ajaji }^{69}\end{array}$ & $\begin{array}{l}\text { Atya-Ugragandha, Moda, Hastimayurka, Khravha, } \\
\text { Kaaravi Valli, Bastamoda, Markat. }\end{array}$ & Apium graveolens var. dulce. \\
\hline Jeeraka & $\begin{array}{l}\text { White=Deerghak, Shuklak, Ajaaji, Kanajeerak. } \\
\text { Black=Jaran Krishna,Varshakaal Sugandhika. }\end{array}$ & $\begin{array}{l}\text { Cuminum cyminum } L \text {. } \\
\text { Carum bulbocastanum/carum } \\
\text { carvi }\end{array}$ \\
\hline Kalounji ${ }^{71}$ & $\begin{array}{c}\text { Kaalika, Vashpika, Kunchi, Kaaravi, Upkunchika, } \\
\text { Prathveeka, Sushsavi, Prathvi, Upkaalika. }\end{array}$ & Nigella sativa $L$. \\
\hline Yavani ${ }^{72}$ & $\begin{array}{c}\text { Yavanika, Yavasaha, Ugragandha, Yavavha, } \\
\text { Bhukadambak. }\end{array}$ & $\begin{array}{l}\text { Carum roxburghianum (DC.) } \\
\text { Craib/trachyspermum ammi/ } \\
\text { sphaeranthus indicus }\end{array}$ \\
\hline Yavaneeya $^{73}$ & Chauhaar, Jantunaashan, Yavaneeya. & Artemisia maritime $L$. \\
\hline Ajagandha ${ }^{74}$ & $\begin{array}{c}\text { Pootikeeta, Varvari, Pootivaravar, Kaarvi, Kharpushpa, } \\
\text { Tungi, Pootimayurak. }\end{array}$ & $\begin{array}{c}\text { Techyspermum roxburghianum } \\
\text { Strobilanthus ciliate (kuriniji or neel } \\
\text { kurinji) }\end{array}$ \\
\hline Parseeka Yavani ${ }^{75}$ & Jawaani, Jawani, Teevra, Tarushka, Madkarini. & Hyocyamus niger $L$. \\
\hline Hingupatri ${ }^{76}$ & $\begin{array}{c}\text { Prathustanvi, Prathvika, Charupatrika, Vaashpika, } \\
\text { Karavi, djoh Tanvi, Bilvika, Deerghika. } \\
\text { Venupatri, Hingushivatika, Jantuka, Raamthi, Naadi, } \\
\text { Pinda, Hinguphala. }\end{array}$ & Ferula jaeschkeana Vatke. \\
\hline Hingu $^{77}$ & $\begin{array}{c}\text { Baahlik, Atyugra, Raamath, Bhootnashan, } \\
\text { Agoodhgandha, Jaran, Jantughna, Soopbhushan. }\end{array}$ & Ferula narthrax Boiss. \\
\hline Sudarshna ${ }^{78}$ & Somvalli, Chakranka, Madhuparnika & $\begin{array}{l}\text { Crinum latifollium/ Tinospora } \\
\text { cordifolia (Thunb.) Miers }\end{array}$ \\
\hline Mayurshikha & Mayuravha, Shikha, Saahsri, Madhukchhada. & $\begin{array}{l}\text { Actiniopteris radiate } \\
\text { Adiantum caudatum } \\
\text { Adiantum dichotoma }\end{array}$ \\
\hline Lakshmana 80 & Putrada, Rakta Bindupatra, Naagini. & $\begin{array}{c}\text { Ipomea marginata/aralia } \\
\text { quinenquefolia }\end{array}$ \\
\hline Chandan $^{81}$ & $\begin{array}{l}\text { Tilaparna, Maharh, Shwetachandan, Bhadrasriya, } \\
\text { Malyaj, Gosheersha, Gandhsarak. }\end{array}$ & Santalum album $L$. \\
\hline Rakta Chandana ${ }^{82}$ & $\begin{array}{l}\text { Uddishta, Lohit, Kshudrachandan, Taamrasar, Raktasar, } \\
\text { Jyoti, Som, Ranajan. }\end{array}$ & Pterocarpus santalinus $L$. \\
\hline Krishna Chandana ${ }^{83}$ & Kaaliyaka, Peetsar, Peeta, Naarayanpriya. & $\begin{array}{l}\text { Santalum paniculatum (hawaiin } \\
\text { sandlewood) }\end{array}$ \\
\hline
\end{tabular}




\begin{tabular}{|c|c|c|}
\hline Karchoor $^{84}$ & Dravid Gandhmoolak, Durlabh Shati & $\begin{array}{l}\text { Hedychium spicatum } \\
\text { Sm. in A.Rees }\end{array}$ \\
\hline Shatimoo ${ }^{85}$ & Palashi, Shadgrantha, Suvrata, Gandhmoolini. & $\begin{array}{l}\text { Hedychium spicatum } \\
\text { Sm. in A.Rees }\end{array}$ \\
\hline Bakula $^{86}$ & Keshar, Madhyagandha, Sinha, Visharad. & Mimusops elengi $L$. \\
\hline $\begin{array}{l}\text { Vush(Vraddha } \\
\text { Molsiri) }\end{array}$ & Vuk, Sthoolpushpa, Vasuka, Shivashodhaka. & Osmanthum fragrans Linn. \\
\hline Udumbara ${ }^{88}$ & $\begin{array}{l}\text { Ksheeravraksha, Jantu-Vraksha, Sadaphala, } \\
\text { Hemadugdha, Yagyaang, Sheetavalkala. }\end{array}$ & Ficus glomerata $L$. \\
\hline Kaak Udumbara ${ }^{89}$ & Falgu, Malaayu, Chitrabheshaj. & Ficus hispida $L$. \\
\hline Plaksha90 & $\begin{array}{l}\text { Plava, Charu-Vraksha, Suparshva, Garbha-Bhaandak } \\
\text { Vati, Kamandal, Yppo, Pippari, Charudarshan. }\end{array}$ & Ficus virens $L$. \\
\hline Nandivraksha ${ }^{91}$ & Ashvaththabheda, Prarohi, Gajapaadap. & $\begin{array}{l}\text { Toona ciliate M. Roem./Ficus } \\
\text { retusa/ Ficus altisimma Blume. }\end{array}$ \\
\hline Vetas $^{92}$ & $\begin{array}{c}\text { Vanjula, Namra Vaneer, Deerghapatraka, Naadeya, } \\
\text { Madhyapushpa, Toyakaam, Nikunjaka. }\end{array}$ & Salix caprea L. \\
\hline Jala Vetasa $a^{93}$ & Jalaukasambhranta, Ambhoj, Nichula. & Salix tetrasperma Roxb. \\
\hline Tirigichhi ${ }^{94}$ & Gajakanta, Karanji, Kshirini, Dvipa. & Caesalpinia crista L. \\
\hline Shami $i^{95}$ & $\begin{array}{l}\text { Tungaa, Shankuphala, Pavitra, Keshhratphala, Lakshmi, } \\
\text { Shivani, Aadhi mati, Bhu-Shami, Shankarahvya. }\end{array}$ & $\begin{array}{l}\text { Prosopis cineraria } \\
\text { (L.) Druce }\end{array}$ \\
\hline Shamishika ${ }^{96}$ & Tinthinika, Durbala, Ambushirishika. & $N A$ \\
\hline Kubjaka ${ }^{97}$ & $\begin{array}{c}\text { Bhadrataruni, Brahtapushpa, Mahasaha, Shatpatri, } \\
\text { Taruni, Karnika, Charukeshara, } \\
\text { Rakta=Raktapushpa, Lakshapushpa, Atimanjula. }\end{array}$ & $\begin{array}{c}\text { Rosa } \times \text { damascene/Rosa centifolia } \\
\text { L. }\end{array}$ \\
\hline Ketakis & $\begin{array}{l}\text { Soochikapushpa, Jambuk, Krakach-Ch-Chhda } \\
\text { Suvarnaketaki=Laghupushpa, Sugandhini. }\end{array}$ & $\begin{array}{l}\text { Costus speciosus/pandanus } \\
\text { odoratissimus }\end{array}$ \\
\hline
\end{tabular}

\section{Discussion}

Madanpal nighantu described by a pattern which is very unique and Most of the drugs have same characteristics; that's why these drugs are placed in a series by the writer of Madanpal Nighantu.

Some of the pattern are giving below:

\section{Absoulate Medicinal Plants}

Author placed the absolute medicinal plants which are more useful in disease; as the name of Abhayadi Varga; So that Some of them are redescribed in other Vargas.

Followed by Gana:

- Triphala DravyasWith Different Amalaki Types.

- Brahtpanchmool Drvayas

- Laghupanchmool Drvyas

- Jeevaniya Gana Drvyas

- Medhya Dravya

- Anti Fertility Dravya

- Chaturabala Plants

Followed by Shodhan and Shaman Dravya:
- Rechan Dravyas

- Vaamak Drvyas

- Aasthapan Dravya

- Kapha Samshaman Drvyas

- Pitta Samshaman Dravya

- Vaatsamshaman Dravya

Followed by Special Davyas:

- Upvisha dravya (Toxic Plants)

- Shaak dravya

- Madkari dravya (Sedative and hypnotic plants)

\section{Followed by Aromatic Plants}

Author placed the aromatic plants in the name of Karpuradi Varga.

\section{Followed by Deepan-Pachan Dravya Plants}

Author placed the digestive plants (as use) in the name of Shunthyadi Varga.

\section{Followed by Fruit Pants}

Author placed the fruity plants in the name of Phaladi Varga. 


\section{Followed by Big Tree Plants}

Author placed the big tree plants in the name of Vatadi Varga.

\section{Followed by Vegetable Plants}

Author placed the vegetable plants in the Shaka Varga.

\section{Conclusion}

Madanpal nighantu or lexicon has a wonderful space in the field of ancient pharmacology. This lexicon is quite nearer of the samhita period and based on nearby ayurvedic medicinal texts. This can be helpful to solve the controversial plants. Basonyms and actions are the only way to find a species of controversial medicinal plant. Madanpal Nighantu put the same morphological characteristic plant or similar species at the same time.

\section{Conflict of Interest: None}

\section{Refrences}

1. Singh AN, Dubey SD. Basics Concepts of Dravyaguna Vijnana, Chaukhambha Vishvabharati, Varanasi. Edition First, 2014: 93.

2. Singh AN, Dubey SD. Basics Concepts Of Dravyaguna Vijnana,Chaukhambha Vishvabharati,Varanasi. Edition First, 2014: 94.

3. http://www.medicinalplants.in/sanskritauthentication.

4. Tipathi PH, Nighantu M, Elaborated With 'Hari' Hindi Commentary; Choukhambha Krishnadas Akadami, Varanasi, Edition 1 15, Abhayadi Varga Verse 61, 2009: 12.

5. Tipathi PH, Nighantu M. Elaborated With 'Hari' Hindi Commentary; Choukhambha Krishnadas Akadami, Varanasi, Edition $1^{\text {st }}$, Abhayadi Varga Verse 63, 2009: 13.

6. Tipathi PH, Nighantu M. Elaborated With 'Hari' Hindi Commentary; Choukhambha Krishnadas Akadami, Varanasi, Edition $1^{\text {st }}$, Abhayadi Varga Verse 88, 2009: 17.

7. Tipathi PH, Nighantu M. Elaborated With 'Hari' Hindi Commentary; Choukhambha Krishnadas Akadami, Varanasi, Edition $1^{\text {st }}$, Abhayadi Varga Verse 89, 2009: 17.

8. Tipathi PH, Nighantu M. Elaborated With 'Hari' Hindi Commentary; Choukhambha Krishnadas Akadami, Varanasi, Edition $1^{\text {st }}$, Abhayadi Varga Verse 224, 2009: 46.

9. Tipathi PH, Nighantu M, Elaborated With 'Hari' Hindi Commentary; Choukhambha Krishnadas Akadami, Varanasi, Edition $1^{\text {st }}$, Abhayadi Varga Verse 74, 2009: 15.

10. Tipathi PH, Nighantu M. Elaborated With 'Hari' Hindi Commentary; Choukhambha Krishnadas Akadami, Varanasi, Edition $1^{\text {st }}$, Abhayadi Varga Verse 75, 2009: 15.

11. Tipathi PH, Nighantu M. Elaborated With 'Hari' Hindi Commentary; Choukhambha Krishnadas Akadami, Varanasi, Edition 1 ${ }^{\text {st }}$, Abhayadi Varga Verse 77, 2009: 15.

12. Tipathi PH, Nighantu M. Elaborated With 'Hari' Hindi Commentary; Choukhambha Krishnadas Akadami,
Varanasi, Edition $1^{\text {st }}$, Abhayadi Varga Verse 78, 2009: 15

13. Tipathi PH, Nighantu M, Elaborated With 'Hari' Hindi Commentary; Choukhambha Krishnadas Akadami, Varanasi, Edition $1^{\text {st }}$, Abhayadi Varga Verse 79, 2009: 16.

14. Tipathi PH, Nighantu M;Elaborated With 'Hari' Hindi Commentary; Choukhambha Krishnadas Akadami, Varanasi, Edition $1^{\text {st }}$, Abhayadi Varga Verse 80, 2009: 16.

15. Tipathi PH, Nighantu M. Elaborated With 'Hari' Hindi Commentary; Choukhambha Krishnadas Akadami, Varanasi, Edition 1 ${ }^{\text {st }}$, Abhayadi Varga Verse 81, 2009: 16.

16. Tipathi PH, Nighantu M, Elaborated With 'Hari' Hindi Commentary; Choukhambha Krishnadas Akadami, Varanasi, Edition 1 ${ }^{\text {st }}$, Abhayadi Varga Verse 82, 2009: 16.

17. PH Tipathi, Nighantu M. Elaborated With 'Hari' Hindi Commentary; Choukhambha Krishnadas Akadami, Varanasi, Edition $1^{\text {st }}$, Abhayadi Varga Verse 93, 2009: 18.

18. Tipathi PH, Nighantu M. Elaborated With 'Hari' Hindi Commentary; Choukhambha Krishnadas Akadami, Varanasi, Edition $1^{\text {st }}$, Abhayadi Varga Verse 94, 2009: 18.

19. Tipathi PH, Nighantu M. Elaborated With 'Hari' Hindi Commentary; Choukhambha Krishnadas Akadami, Varanasi, Edition 1 ${ }^{\text {st }}$, Abhayadi Varga Verse 97, 2009: 19.

20. Tipathi PH, Nighantu M. Elaborated With 'Hari' Hindi Commentary; Choukhambha Krishnadas Akadami, Varanasi, Edition $1^{\text {st }}$, Abhayadi Varga Verse 98, 2009: 19.

21. Tipathi PH, Nighantu M. Elaborated With 'Hari' Hindi Commentary; Choukhambha Krishnadas Akadami, Varanasi, Edition $1^{\text {st }}$, Abhayadi Varga Verse 100, 2009: 20.

22. Tipathi PH, Nighantu M. Elaborated With 'Hari' Hindi Commentary; Choukhambha Krishnadas Akadami, Varanasi, Edition $1^{\text {st }}$, Abhayadi Varga Verse 101, 2009: 20.

23. Tipathi PH, Nighantu M. Elaborated With 'Hari' Hindi Commentary; Choukhambha Krishnadas Akadami, Varanasi, Edition $1^{\text {st }}$, Abhayadi Varga Verse 101, 2009: 20.

24. Tipathi PH, Nighantu M. Elaborated With 'Hari' Hindi Commentary; Choukhambha Krishnadas Akadami, Varanasi, Edition $1^{\text {st }}$, Abhayadi Varga Verse 106, 2009: 21.

25. Tipathi PH, Nighantu M. Elaborated With 'Hari' Hindi Commentary; Choukhambha Krishnadas Akadami, Varanasi, Edition $1^{\text {st }}$, Abhayadi Varga Verse 108, 2009: 22.

26. Tipathi PH; Nighantu M. Elaborated With 'Hari' Hindi Commentary; Choukhambha Krishnadas Akadami,Varanasi,Edition $1^{\text {st }}$, Abhayadi Varga Verse 111-112, 2009: 23.

27. Tipathi PH, Nighantu M. Elaborated With 'Hari' Hindi Commentary; Choukhambha Krishnadas Akadami, Varanasi, Edition $1^{\text {st }}$, Abhayadi Varga Verse 114, 2009: 23 . 
28. Tipathi PH, Nighantu M. Elaborated With 'Hari' Hindi Commentary; Choukhambha Krishnadas Akadami, Varanasi, Edition $1^{\text {st }}$, Abhayadi Varga Verse 115, 2009: 23.

29. Tipathi PH, Nighantu M. Elaborated With 'Hari' Hindi Commentary; Choukhambha Krishnadas Akadami, Varanasi, Edition $1^{\text {st }}$, Abhayadi Varga Verse 117, 2009: 24.

30. Tipathi PH, Nighantu M. Elaborated With 'Hari' Hindi Commentary; Choukhambha Krishnadas Akadami, Varanasi, Edition $1^{\text {st }}$, Abhayadi Varga Verse 119, 2009: 24.

31. Tipathi PH, Nighantu M. Elaborated With 'Hari' Hindi Commentary; Choukhambha Krishnadas Akadami, Varanasi, Edition $1^{\text {st }}$, Abhayadi Varga Verse 120-121, 2009: 24

32. Tipathi PH, Nighantu M. Elaborated With 'Hari' Hindi Commentary; Choukhambha Krishnadas Akadami, Varanasi, Edition $1^{\text {st }}$, Abhayadi Varga Verse 135, 2009 : 28.

33. Tipathi PH, Nighantu M. Elaborated With 'Hari' Hindi Commentary; Choukhambha Krishnadas Akadami, Varanasi, Edition $1^{\text {st }}$, Abhayadi Varga Verse 153, 2009 : 32.

34. Tipathi PH, Nighantu M. Elaborated With 'Hari' Hindi Commentary; Choukhambha Krishnadas Akadami, Varanasi, Edition $1^{\text {st }}$, Abhayadi Varga Verse 155, 2009: 32.

35. Tipathi PH, Nighantu M. Elaborated With 'Hari’ Hindi Commentary; Choukhambha Krishnadas Akadami, Varanasi, Edition $1^{\text {st }}$, Abhayadi Varga Verse 143, 2009 : 29.

36. Tipathi PH, Nighantu M. Elaborated With 'Hari' Hindi Commentary; Choukhambha Krishnadas Akadami, Varanasi, Edition $1^{\text {st }}$, Abhayadi Varga Verse 244, 2009 : 50.

37. Tipathi PH, Nighantu M. Elaborated With 'Hari' Hindi Commentary; Choukhambha Krishnadas Akadami, Varanasi, Edition $1^{\text {st }}$, Abhayadi Varga Verse 157, 2009 : 33.

38. Tipathi PH, Nighantu M. Elaborated With 'Hari' Hindi Commentary; Choukhambha Krishnadas Akadami, Varanasi, Edition $1^{\text {st }}$, Abhayadi Varga Verse 159, 2009 : 33.

39. Tipathi PH, Nighantu M. Elaborated With 'Hari' Hindi Commentary; Choukhambha Krishnadas Akadami, Varanasi, Edition $1^{\text {st }}$, Abhayadi Varga Verse 162, 2009: 34.

40. Tipathi PH, Nighantu M. Elaborated With 'Hari' Hindi Commentary; Choukhambha Krishnadas Akadami, Varanasi, Edition $1^{\text {st }}$, Abhayadi Varga Verse 177, 2009: 37 .

41. Tipathi PH, Nighantu M. Elaborated With 'Hari' Hindi
Commentary; Choukhambha Krishnadas Akadami, Varanasi, Edition $1^{\text {st }}$, Abhayadi Varga Verse 179, 2009: 37.

42. Tipathi PH, Nighantu M. Elaborated With 'Hari' Hindi Commentary; Choukhambha Krishnadas Akadami, Varanasi, Edition $1^{\text {st }}$, Abhayadi Varga Verse 182, 2009: 38.

43. Tipathi PH, Nighantu M. Elaborated With 'Hari' Hindi Commentary; Choukhambha Krishnadas Akadami, Varanasi, Edition $1^{\text {st }}$, Abhayadi Varga Verse 183, 2009 : 38.

44. Tipathi PH, Nighantu M. Elaborated With 'Hari' Hindi Commentary; Choukhambha Krishnadas Akadami, Varanasi, Edition $1^{\text {st }}$, Abhayadi Varga Verse 184, 2009: 38.

45. Tipathi PH, Nighantu M. Elaborated With 'Hari' Hindi Commentary; Choukhambha Krishnadas Akadami, Varanasi, Edition $1^{\text {st }}$, Abhayadi Varga Verse 185, 2009 : 38.

46. Tipathi PH, Nighantu M. Elaborated With 'Hari' Hindi Commentary; Choukhambha Krishnadas Akadami, Varanasi, Edition $1^{\text {st }}$, Abhayadi Varga Verse 175, 2009: 36.

47. Tipathi PH, Nighantu M. Elaborated With 'Hari' Hindi Commentary; Choukhambha Krishnadas Akadami, Varanasi, Edition $1^{\text {st }}$, Abhayadi Varga Verse 217, 2009: 45.

48. Tipathi PH, Nighantu M. Elaborated With 'Hari' Hindi Commentary; Choukhambha Krishnadas Akadami, Varanasi, Edition ${ }^{\text {st }}$, Abhayadi Varga Verse 218, 2009 : 45.

49. Tipathi PH, Nighantu M. Elaborated With 'Hari' Hindi Commentary; Choukhambha Krishnadas Akadami, Varanasi, Edition $1^{\text {st }}$, Abhayadi Varga Verse 261, 2009: 54.

50. Tipathi PH, Nighantu M. Elaborated With 'Hari' Hindi Commentary; Choukhambha Krishnadas Akadami, Varanasi, Edition $1^{\text {st }}$, Abhayadi Varga Verse 262, 2009: 54.

51. Tipathi PH, Nighantu M. Elaborated With 'Hari' Hindi Commentary; Choukhambha Krishnadas Akadami, Varanasi, Edition $1^{\text {st }}$, Abhayadi Varga Verse 265, 2009: 55.

52. Tipathi PH, Nighantu M. Elaborated With 'Hari' Hindi Commentary; Choukhambha Krishnadas Akadami, Varanasi, Edition $1^{\text {st }}$, Abhayadi Varga Verse 266, 2009; 55.

53. Tipathi PH, Nighantu M. Elaborated With 'Hari' Hindi Commentary; Choukhambha Krishnadas Akadami, Varanasi, Edition $1^{\text {st }}$, Abhayadi Varga Verse 268, 2009: 56.

54. Tipathi PH, Nighantu M. Elaborated With 'Hari' Hindi Commentary; Choukhambha Krishnadas Akadami, 
Varanasi, Edition $1^{\text {st }}$, Abhayadi Varga Verse 285, 2009: 60.

55. Tipathi PH, Nighantu M. Elaborated With 'Hari' Hindi Commentary; Choukhambha Krishnadas Akadami, Varanasi, Edition $1^{\text {st }}, 2009$ : Abhayadi Varga Verse 285, 60.

56. Tipathi PH, Nighantu M. Elaborated With 'Hari' Hindi Commentary; Choukhambha Krishnadas Akadami, Varanasi, Edition $1^{\text {st }}$, Abhayadi Varga Verse 313, 2009: 67.

57. Tipathi PH, Nighantu M. Elaborated With 'Hari' Hindi Commentary; Choukhambha Krishnadas Akadami, Varanasi, Edition $1^{\text {st }}$, Abhayadi Varga Verse 293, 2009: 62.

58. Tipathi PH, Nighantu M. Elaborated With 'Hari' Hindi Commentary; Choukhambha Krishnadas Akadami, Varanasi, Edition $1^{\text {st }}$, Abhayadi Varga Verse 297, 2009: 63.

59. Tipathi PH, Nighantu M. Elaborated With 'Hari' Hindi Commentary; Choukhambha Krishnadas Akadami, Varanasi, Edition $1^{\text {st }}$, Abhayadi Varga Verse 275, 2009: 57.

60. Tipathi PH, Nighantu M. Elaborated With 'Hari' Hindi Commentary; Choukhambha Krishnadas Akadami, Varanasi, Edition $1^{\text {st }}$, Abhayadi Varga Verse 311, 2009: 66.

61. Tipathi PH, Nighantu M. Elaborated With 'Hari' Hindi Commentary; Choukhambha Krishnadas Akadami, Varanasi, Edition $1^{\text {st }}$, Abhayadi Varga Verse 312, 2009 : 66.

62. Tipathi PH, Nighantu M. Elaborated With 'Hari' Hindi Commentary; Choukhambha Krishnadas Akadami, Varanasi, Edition $1^{\text {st }}$, Abhayadi Varga Verse 335, 2009: 72.

63. Tipathi PH, Nighantu M. Elaborated With 'Hari' Hindi Commentary; Choukhambha Krishnadas Akadami, Varanasi, Edition $1^{\text {st }}$, Abhayadi Varga Verse 290/336, 2009; 61-72.

64. Tipathi PH, Nighantu M. Elaborated With 'Hari' Hindi Commentary; Choukhambha Krishnadas Akadami, Varanasi, Edition $1^{\text {st }}$, Abhayadi Varga Verse 291, 2009: 61.

65. Tipathi PH, Nighantu M. Elaborated With 'Hari' Hindi Commentary; Choukhambha Krishnadas Akadami, Varanasi, Edition $1^{\text {st }}$, Shunthyadi Varga Verse 18, 2009: 79.

66. Tipathi PH, Nighantu M. Elaborated With 'Hari' Hindi Commentary; Choukhambha Krishnadas Akadami, Varanasi, Edition $1^{\text {st }}$, Shunthyadi Varga Verse 19, 2009: 79.

67. Tipathi PH, Nighantu M. Elaborated With 'Hari' Hindi Commentary; Choukhambha Krishnadas Akadami, Varanasi, Edition $1^{\text {st }}$, Shunthyadi Varga Verse 23, 2009:
80.

68. Tipathi PH, Nighantu M. Elaborated With 'Hari' Hindi Commentary; Choukhambha Krishnadas Akadami, Varanasi, Edition $1^{\text {st }}$, Shunthyadi Varga Verse 25, 2009; 80.

69. Tipathi PH, Nighantu M. Elaborated With 'Hari' Hindi Commentary; Choukhambha Krishnadas Akadami, Varanasi, Edition $1^{\text {st }}$, Shunthyadi Varga Verse 27, 2009: 81.

70. Tipathi PH, Nighantu M. Elaborated With 'Hari' Hindi Commentary; Choukhambha Krishnadas Akadami, Varanasi, Edition $1^{\text {st }}$, Shunthyadi Varga Verse 29, 2009; 81.

71. Tipathi PH, Nighantu M. Elaborated With 'Hari' Hindi Commentary; Choukhambha Krishnadas Akadami, Varanasi, Edition $1^{\text {st }}$, Shunthyadi Varga Verse 30, 2009; 81.

72. Tipathi PH, Madanpal Nighantu. Elaborated With 'Hari' Hindi Commentary; Choukhambha Krishnadas Akadami,Varanasi,Edition $1^{\text {st }}$, Shunthyadi Varga Verse 32, 2009: 82.

73. Tipathi PH, Nighantu M. Elaborated With 'Hari' Hindi Commentary; Choukhambha Krishnadas Akadami, Varanasi, Edition $1^{\text {st }}$, Shunthyadi Varga Verse 34, 2009: 82.

74. Tipathi PH, Nighantu M. Elaborated With 'Hari' Hindi Commentary; Choukhambha Krishnadas Akadami, Varanasi, Edition $1^{\text {st }}$, Shunthyadi Varga Verse 303/35, 2009: 64/82.

75. Tipathi PH, Nighantu M. Elaborated With 'Hari' Hindi Commentary; Choukhambha Krishnadas Akadami, Varanasi, Edition $1^{\text {st }}$, Shunthyadi Varga Verse 343, 2009: 74.

76. Tipathi PH, Nighantu M. Elaborated With 'Hari' Hindi Commentary; Choukhambha Krishnadas Akadami, Varanasi, Edition $1^{\text {st }}$, Shunthyadi Varga Verse 46-47, 2009: 84.

77. Tipathi PH, Nighantu M. Elaborated With 'Hari' Hindi Commentary; Choukhambha Krishnadas Akadami, Varanasi, Edition $1^{\text {st }}$, Shunthyadi Varga Verse 49, 2009: 84.

78. Tipathi PH, NighantuM. Elaborated With 'Hari' Hindi Commentary; Choukhambha Krishnadas Akadami, Varanasi, Edition $1^{\text {st }}$, Shunthyadi Varga Verse 316, 2009: 67.

79. Tipathi PH, Nighantu M. Elaborated With 'Hari' Hindi Commentary; Choukhambha Krishnadas Akadami, Varanasi, Edition $1^{\text {st }}$, Shunthyadi Varga Verse 317, 2009: 68.

80. Tipathi $\mathrm{PH}$, Nighantu M. Elaborated With 'Hari' Hindi Commentary; Choukhambha Krishnadas Akadami, Varanasi, Edition $1^{\text {st }}$,Shunthyadi Varga Verse 318, 2009 : 68. 
81. Tipathi PH, Nighantu M. Elaborated With 'Hari' Hindi Commentary; Choukhambha Krishnadas Akadami, Varanasi, Edition $1^{\text {st }}$, Karpuradi Varga Verse 07, 2009: 91.

82. Tipathi $\mathrm{PH}$, Nighantu M. Elaborated With 'Hari' Hindi Commentary; Choukhambha Krishnadas Akadami, Varanasi, Edition $1^{\text {st }}$, Karpuradi Varga Verse 09, 2009: 91.

83. Tipathi PH, Nighantu M. Elaborated With 'Hari' Hindi Commentary; Choukhambha Krishnadas Akadami, Varanasi, Edition $1^{\text {st }}$, Karpuradi Varga Verse 11, 2009: 91.

84. Tipathi PH, Nighantu M. Elaborated With 'Hari' Hindi Commentary; Choukhambha Krishnadas Akadami, Varanasi, Edition $1^{\text {st }}$, Karpuradi Varga Verse 54, 2009 : 101.

85. Tipathi PH, Nighantu M. Elaborated With 'Hari' Hindi Commentary; Choukhambha Krishnadas Akadami, Varanasi, Edition $1^{\text {st }}$, Karpuradi Varga Verse 55, 2009: 101.

86. Tipathi PH, Nighantu M. Elaborated With 'Hari' Hindi Commentary; Choukhambha Krishnadas Akadami, Varanasi, Edition $1^{\text {st }}$, Karpuradi Varga Verse 96, 2009 : 111.

87. Tipathi PH, Nighantu M. Elaborated With 'Hari' Hindi Commentary; Choukhambha Krishnadas Akadami, Varanasi, Edition $1^{\text {st }}$, Karpuradi Varga Verse 97, 2009: 112.

88. Tipathi PH, Nighantu M. Elaborated With 'Hari' Hindi Commentary; Choukhambha Krishnadas Akadami, Varanasi, Edition $1^{\text {st }}$, Vatadi Varga Verse 04, 2009: 130.

89. Tipathi PH, Nighantu M. Elaborated With 'Hari' Hindi Commentary; Choukhambha Krishnadas Akadami, Varanasi, Edition $1^{\text {st }}$, Vatadi Varga Verse 05, 2009: 130.

90. Tipathi PH, Nighantu M. Elaborated With 'Hari' Hindi Commentary; Choukhambha Krishnadas Akadami, Varanasi, Edition 1 ${ }^{\text {st }}$, Vatadi Varga Verse 06, 2009: 130.

91. Tipathi PH, Nighantu M. Elaborated With 'Hari' Hindi Commentary; Choukhambha Krishnadas Akadami, Varanasi, Edition $1^{\text {st }}$, Vatadi Varga Verse 11, 2009: 131.

92. Tipathi PH, Nighantu M. Elaborated With 'Hari' Hindi Commentary; Choukhambha Krishnadas Akadami, Varanasi, Edition $1^{\text {st }}$, Vatadi Varga Verse 16, 2009: 113.

93. Tipathi PH, Nighantu M. Elaborated With 'Hari' Hindi Commentary; Choukhambha Krishnadas Akadami, Varanasi, Edition $1^{\text {st }}$, Vatadi Varga Verse 17, 2009: 113.

94. Tipathi PH, Nighantu M. Elaborated With 'Hari' Hindi Commentary; Choukhambha Krishnadas Akadami, Varanasi, Edition $1^{\text {st }}$, Vatadi Varga Verse 18, 2009: 113.

95. Tipathi PH, Nighantu M. Elaborated With 'Hari' Hindi Commentary; Choukhambha Krishnadas Akadami, Varanasi, Edition $1^{\text {st }}$, Vatadi Varga Verse 63, 2009: 143.

96. Tipathi PH, Nighantu M. Elaborated With 'Hari' Hindi Commentary; Choukhambha Krishnadas Akadami, Varanasi, Edition $1^{\text {st }}$, Vatadi Varga Verse 63, 2009: 143.
97. Tipathi PH, Nighantu M. Elaborated With 'Hari' Hindi Commentary; Choukhambha Krishnadas Akadami, Varanasi, Edition $1^{\text {st }}$, Karpuradi Varga Verse 109, 2009: 87.

98. Tipathi PH, Nighantu M. Elaborated With 'Hari' Hindi Commentary; Choukhambha Krishnadas Akadami, Varanasi, Edition $1^{\text {st }}$, Karpuradi Varga Verse 110, 2009: 89. 